\title{
La conformación histórica de una región
}

\author{
Luis Rodríguez Castillo•
}

doi: http://dx.doi.org/10.32870/espiral.v25i72.6040.g6075

En contraste con las publicaciones recientes sobre el estado de Michoacán, que enfatizan la ausencia del Estado y la generalización de la violencia, este libro aborda, por su parte, a la región Ciénega de Michoacán, no ajena a la violencia, pero en la que "el Estado ha tenido una presencia histórica significativa” (p. 9), lo que ha implicado grandes inversiones y la intervención de múltiples agentes en disputa por el control de recursos.

La noción de región en este libro está comprometida con las que retoman la profundidad histórica como factor explicativo, particularmente con Andrés Fábregas, Guillermo de la Peña y Eric van Young, para quienes las relaciones sociales que conforman una región sólo pueden comprenderse como un resultado histórico-cultural. Y sobre la importancia de las relaciones de poder en los procesos sociales, el libro retoma las posturas de William Roseberry y Eric Wolf.

No obstante, y en lo que podríamos caracterizar como una perspectiva de antropología económica y del estudio de las nuevas ruralidades, el libro se propone estudiar cuatro "procesos socio-históricos que han moldeado a la región y su actividad produc-

Guillermo Paleta Pérez (2016). La conformación histórica de una región: desecación y transformaciones rurales en la ciénega de Chapala, Michoacán. México: Ciudad Universitaria, Unidad Académica de Estudios Regionales, Coordinación de Humanidades, Universidad Nacional Autónoma de México. 
tiva" (p. 10), así como las trasformaciones de los dos centros urbanos principales de la región: Jiquilpan y Sahuayo:

a) el establecimiento de las haciendas agropecuarias en la ciénega y la exclusión social; b) la desecación de la laguna de Chapala y la configuración de la región;c) la conformación del distrito de riego 024;y d) el proceso de reconversión productiva en la región (p. I3).

Dicho estudio es abordado en este libro a través de seis capítulos y conclusiones.

En el primer capítulo, "La ciénega de Chapala, Michoacán, y los estudios regionales", se aborda un estado de la cuestión sobre la región como categoría analítica, de lo que el autor concluye que esta "es apropiada de manera diferenciada por los actores sociales en contextos históricos específicos y en constante negociación con hegemonías locales" (p. 18). También, se aborda el contexto de la ciénega michoacana.

"La configuración de la región y su paisaje rural", título del segundo acápite, describe los antecedentes lacustres de la ciénega y de su paisaje rural. El capítulo se adhiere para ello a la noción de modo de vida lacustre, pero destaca que este fue un modo de vida que se fue perdiendo conforme se transformaron los paisajes, acorde a los intereses prevalecientes. Concluye que en ese proceso histórico, estas diferencias intrarregionales "radican en el control e intermediación de productos agropecuarios de la zona y fuera de ella, así como también por límites territoriales" (p. 51).

El tercer capítulo, "La hacienda y la conformación de estructuras sociales de exclusión en la ciénega de Chapala", plantea que la región se caracterizaría "hasta finales del siglo XVIII [...] por el predominio del sistema de haciendas y el control de la tierra y el agua" (p. 59). Constata como válida la hipótesis de Boehm de que, como resultado del proceso modernizador y la pujante productividad en la 
región, los hacendados de la ciénega contrajeron deudas impagables que los obligarían a dejar el manejo del agua y ceder sus tierras a los actores emergentes del cardenismo: los ejidatarios.

El cuarto acápite, "El proceso de desecación, cambio social y el entorno regional”, argumenta que la desecación en la ciénega michoacana generó cambios en la reorganización territorial y en maneras específicas de explotación de los recursos naturales. Analiza cómo se van creando mecanismos para la centralización del control de esos recursos y cierra con una interesante descripción de la infraestructura del distrito de riego 024, ciénega de Chapala, Michoacán.

"Transformaciones rurales. La reconversión productiva", el quinto capítulo, aborda las transformaciones recientes en la región a partir de la emisión de la Ley de Desarrollo Rural Sustentable, de 2001. Además de describir las transformaciones organizativas y las características del distrito 089 Sahuayo, en el que "existen cinco cadenas productivas que son: maíz, hortalizas, sorgo, pesca y ganado bovino doble propósito" (p. 101), describe las actividades agrícolas en los municipios de Jiquilpan y Sahuayo. No obstante, el cambio de uso de suelo es un aspecto de relevancia que también es abordado y del que se transcribe una cita larga, pero necesaria:

En poco tiempo los límites geográficos entre Jiquilpan y Sahuayo desaparecerán. Estas ciudades a pesar de su crecimiento mantienen características totalmente rurales, me refiero a su dinámica y organización social basada en el sistema de parentesco, el orden religioso y las relaciones de confianza. Llama la atención que ambas ciudades tienen dinámicas rurales, empero, con problemáticas totalmente de ciudad: caos vehicular, comercio desbordado, crecimiento sin orden, violencia, narcotráfico, corrupción y servicios de mala calidad (p. I 17). 
Sin duda alguna, aquí encontramos no sólo la descripción de los efectos del acelerado proceso de urbanización, sino también los elementos emergentes para una nueva agenda de investigación regional.

En el capítulo sexto, "La producción de agave tequilero en la ciénega”, se evidencia no sólo el proceso de reconversión productiva, sino también la persistencia e importancia de las actividades agrícolas en la región y, particularmente, en torno a un cultivo: el agave, pero también que fue en el periodo de Gobierno de Tinoco Rubí (1996-2002) cuando se promovió la obtención del reconocimiento de la denominación de origen del tequila, que se lograría en 2005 para treinta municipios michoacanos, de los cuales trece pertenecen a la región de estudio, con resultados desde luego, disparejos.

En las conclusiones, finalmente, el autor recapitula los procesos principales que se detonan a partir de la desecación del lago de Chapala, entre otros: las afectaciones a las comunidades ribereñas, la restricción de acceso a recursos a indígenas y campesinos, la especialización agroganadera en el pasado, las disputas por límites municipales, y la reconversión productiva en fechas más recientes, para aseverar que son estos procesos situados en una visión de larga data los que permiten comprender cómo se configura una región mexicana.

En primer lugar, este libro, aunque contiene datos históricos y el autor hace explícito su apego a la historia, no está confeccionado a la manera del historiador que busca identificar los flujos y secuencias temporales o cronológicas. En cada uno de los capítulos, el lector se encontrará en el ir y venir entre los hechos del pasado y el presente, en un ejercicio innovador del autor que le permite identificar rupturas y continuidades en torno a un tópico en particular.

En segundo lugar, la multiplicidad de fuentes es algo muy relevante de este trabajo. En la confección de cada uno 
de los capítulos, el autor echa mano lo mismo de fuentes históricas que de observaciones propias elaboradas sobre el terreno, lo mismo de la revisión de estudios e investigaciones previas que de las entrevistas a profundidad, lo mismo del dato duro, estadístico, que de los decires locales sobre aquello que da identidad a la región.

Metodológicamente, los dos primeros capítulos están comprometidos, si es que estamos interpretando correctamente al autor, con la propuesta de Ángel Palerm para el estudio de la evolución multilineal de los paisajes antropológicos, y la de Thierry Linck para el estudio de los paisajes rurales. Por su parte, el tercero y cuarto capítulos se encuentran mucho más comprometidos con el análisis de estudios y fuentes históricas. En el quinto y sexto capítulos, podría señalarse que el autor se compromete con lo que el antropólogo Alban Bensa denomina conceptualización baja, es decir, aquella estrategia que permite comprender a los actores a través de su propia expresión, y que, por lo tanto, es la vía para evitar, evidentemente, muchas derivas y aspavientos teóricos.

No obstante, existen tres aspectos a destacar que, al estar ausentes en este trabajo, resultan en una invitación a asumirlos de manera crítica en las investigaciones en curso y futuras publicaciones del autor:

1) Si bien en el libro se hace un magnifico recuento y caracterización de lo que es la ciénega michoacana a través de los principales académicos que la han estudiado, se extraña una discusión más densa sobre los límites de las miradas anteriores y del aporte propio del autor.

2) Se enfatiza sobre las ideas que se construyen sobre los espacios, pero un tema sobre el que aún falta profundizar es el de las fronteras y las regiones imaginarias o, por la alusión a la identidad en las conclusiones, la región misma como una representación cultural.

3) Al mirar desde el presente etnográfico, es de interés preguntarse por la configuración que resulta de los procesos 
de conurbanización en Jiquilpan-Sahuayo. Entonces, a su mirada especializada desde las nuevas ruralidades, será necesario nutrirla con las teorías del urbanismo y del desarrollo de políticas públicas en torno a este caso.

En cuanto a la cuestión teórica, son adecuadas las discusiones que se invocan sobre territorio, territorialidad, región, paisaje; no obstante, poco se discute el asunto de categorías como la territorialidad y de los paisajes como herramientas analíticas. En ese mismo sentido, el autor señala que "esta zona era una región de frontera" (p. 61), pero no realiza una discusión teórica sobre esta categoría y sobre la frontera como límite y como espacio de expansión, ambos fenómenos vividos en la región Lerma-Chapala.

Otra arista es lo metodológico, es decir, la vinculación o uso de las categorías para el análisis de los datos positivos y la aceptación o rechazo de las hipótesis. Sobre la confirmación de su primera hipótesis no hay dudas, pero hubiese sido conveniente una reflexión mayor en las conclusiones en relación con la segunda. En ese mismo tenor, la invitación es a recuperar una visión crítica sobre el para qué de la construcción de un marco teórico y su adecuado uso a lo largo de nuestras narrativas y textos, pues buena parte de las categorías que se discuten se diluyen en la presentación de los capítulos.

El escritor hace múltiples llamados a través de sus autores seleccionados, tanto en el ámbito teórico como en lo que respecta al estudio específico de la ciénega michoacana, a entender la región como resultado sociohistórico de relaciones de poder. No obstante, el lector no debe esperar un trabajo que abunde sobre la política, lo político y el poder como ejes explicativos de la constitución de la región.

Este libro resulta de gran interés no sólo para los especialistas de la región de la ciénega. Por la articulación de sus discusiones y temáticas, debe ser lectura imprescindible 
para los estudiosos de las regiones y de la nueva ruralidad, porque es posible encontrar dos objetivos teóricos del material que resultan fundamentales:

- Aportar elementos de discusión acerca de la formación regional a través de la intervención del Estado.

- Generar miradas analíticas en las cuales la interdisciplinariedad puede arrojar resultados provechosos para la comprensión de la realidad social contemporánea.

Aunado a lo anterior, por la presentación de datos históricos y estadísticos, que revelan el prolijo trabajo de un académico consolidado, el libro es de utilidad para los tomadores de decisiones interesados en tener una perspectiva del devenir histórico, así como un diagnóstico claro de la situación que guardan la región y municipalidades estudiadas.

Para un público amplio, debe ser leído no sólo porque en sus páginas encontramos un esfuerzo de microhistoria al más puro estilo gonzaliano, sino porque el lector encontrará una minucia de observaciones del autor, de viajeros y de historiadores, así como un anecdotario cultural (a veces chusco, otras trágico) que marca las diferencias entre santurrones y liberales, entre comerciantes y agroganaderos, y muchas otras, que hacen a Jiquilpan y a Sahuayo ser lo que son, con su sabor particular culturalmente distintivo, y no otra cosa.

Al final del camino, la aportación central de este libro se encuentra en que región y Estado no son presentados como dos entes homogéneos que se contraponen: son grupos, élites y personas de carne y hueso que para su sobrevivencia y la de sus formas de vida entran en luchas por el control de los recursos productivos, el proceso de trabajo, así como disputas por la producción discursiva y las formaciones ideológicas que conforman la conciencia de los sujetos que anclan su comprensión del mundo en el ámbito rural. Una discusión sobre la cual, aunque la cosecha sea a veces cenceña, en las ciencias sociales se tiene aún mucho por trillar. 